\title{
SOME REMARKS ON ELASTIC CRACK-TIP STRESS FIELDS
}

\author{
JAMES R. RICE \\ Division of Engineering, Brown University, Providence, Rhode Island
}

\begin{abstract}
It is shown that if the displacement field and stress intensity factor are known as functions of crack length for any symmetrical load system acting on a linear elastic body in plane strain, then the stress intensity factor for any other symmetrical load system whatsoever on the same body may be directly determined. The result is closely related to Bueckner's [1] "weight function", through which the stress intensity factor is expressed as a sum of work-like products between applied forces and values of the weight function at their points of application. An example of the method is given wherein the solution for a crack in a remotely uniform stress feld is used to generate the expression for the stress intensity factor due to an arbitrary traction distribution on the faces of a crack. A corresponding theory is developed in an appendix for three-dimensional crack problems, although this appears to be directly useful chiefly for problems in which there is axial symmetry.
\end{abstract}

\section{INTRODUCTION}

CONSIDER a two-dimensional linear elastic body containing a straight crack under conditions of plane strain or of generalized plane stress. Both the body and all applied load systems to be considered are assumed symmetrical about the crack line so that only the tensile opening mode of crack tip deformation may result. Two distinct load systems are shown in Fig. 1 and denoted by $Q_{1}$ and $Q_{2}$. The displacement field and stress intensity factor are assumed known as a function of crack length $l$ for one of the load systems, say $Q_{1}$.

The principal result of this study is in showing that this information is sufficient to determine the stress intensity factor for the other load system $Q_{2}$. Of course, the 1 and 2 systems may represent any arbitrarily chosen load systems and thus it is being shown that if a solution for the displacement field and stress intensity factor is known for any particular load system, then this information is sufficient to determine the stress intensity factor for any other load system whatsoever.

Bueckner [1] has presented a similar result, basing his argument on analytic function representations of elastic fields for isotropic materials. Here we see that this dependence between solutions for different load systems arises as a consequence of what is known on the relation between stress intensity factors and strain energy variations $[2,3]$ and of the properties of point functions. To develop the argument, consider the following preliminary remarks:

(a) $Q_{1}$ and $Q_{2}$ are considered as "generalized forces" in the sense that the stress vector $\mathbf{t}$ on the boundary $\Gamma$ and body force $f$ within region $A$ resulting from, say, load system 1 are expressible in the form

$$
\mathbf{t}=Q_{1} \mathbf{t}^{(1)} \text { on } \Gamma \quad \text { and } \mathbf{f}=Q_{1} \mathbf{f}^{(1)} \quad \text { in } A \text {, }
$$




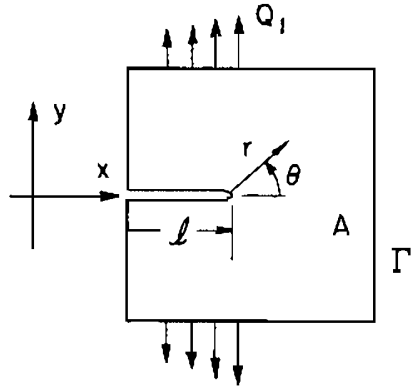

LOAD SYSTEM "I"

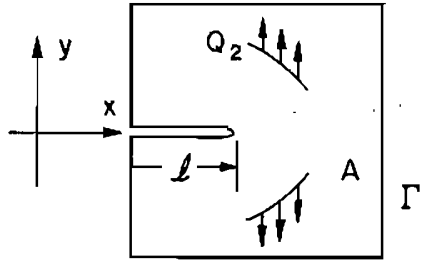

LOAD SYSTEM " $2 "$

FIG. 1.

with similar expressions for load system 2, where $\mathbf{t}^{(1)}, \mathbf{t}^{(2)}$ and $\mathbf{f}^{(1)}, \mathbf{f}^{(2)}$ are functions of position only.

(b) If $\mathbf{u}$ is any displacement field in the body, then with it we may associate "generalized displacements" $q_{1}$ and $q_{2}$ by

$$
q_{i}=\int_{\Gamma} \mathbf{t}^{(i)} \cdot \mathbf{u} \mathrm{d} \Gamma+\int_{A} \mathbf{f}^{(i)} \cdot \mathbf{u} \mathrm{d} A .
$$

Thus if a variation $\delta \mathbf{u}$ is given to the displacement field, $Q_{1} \delta q_{1}$ will be the work (per unit thickness) of load system 1 and $Q_{2} \delta q_{2}$ of 2 . We shall write $Q_{1} \mathbf{u}^{(1)}$ for the elastic displacement field induced by load system 1 and $Q_{2} \mathbf{u}^{(2)}$ for that by 2 . Hence if both load systems are simultaneously applied to the body, then by superposition

$$
\mathbf{u}=Q_{1} \mathbf{u}^{(1)}+Q_{2} \mathbf{u}^{(2)} \text { and } q_{i}=C_{i j} Q_{j}
$$

(summing on repeated indices) where the compliances are

$$
C_{i j}=\int_{\Gamma} \mathbf{t}^{(i)} \cdot \mathbf{u}^{(j)} \mathrm{d} \Gamma+\int_{A} \mathbf{f}^{(i)} \cdot \mathbf{u}^{(j)} \mathrm{d} A
$$

Here $C_{i j}=C_{i j}(l)$ because $\mathbf{u}^{(i)}=\mathbf{u}^{(i)}(x, y, l)$.

(c) The stress intensity factor $K$ is defined by

$$
K=\lim _{r \rightarrow 0}(2 \pi r)^{\frac{1}{2}} \sigma_{y y},
$$

where $\sigma_{y y}$ is the $y$ directed tensile stress acting at distance $r$ along the line directly ahead of the crack tip. We denote $Q_{1} K^{(1)}$ as the factor induced by load system 1 and $Q_{2} K^{(2)}$ as that by 2 , where $K^{(i)}=K^{(i)}(l)$.

(d) It is known that if $W$ is the elastic strain energy (per unit thickness) of a loaded, cracked body, then

$$
(\partial W / \partial l)_{\text {fixed displ. }}=-K^{2} / H
$$

where "fixed displacements" means that the derivative is taken under conditions for which loaded portions of the body are constrained against working displacements. $H$ is an appropriate elastic modulus : for an isotropic material it is $E / 1-v^{2}$ for plane strain and $E$ for generalized plane stress; for anisotropic materials the modulus may be chosen from 
the work of Sih et al. [4]. Thus when both load systems are simultaneously applied to the same body, we may think of representing $W$ as a function of $q_{1}, q_{2}$ and $l$, and write

$$
\partial W\left(q_{1}, q_{2}, l\right) / \partial l=-K^{2} / H, \text { where } K=Q_{1} K^{(1)}(l)+Q_{2} K^{(2)}(l) .
$$

Of course, $\partial W\left(q_{1}, q_{2}, l\right) / \partial q_{i}=Q_{i}$.

These last remarks enable us to write, in the case of simultaneous action of both load systems, the perfect Pfaffian differential form

$$
Q_{1} \delta q_{1}+Q_{2} \delta q_{2}-\left(K^{2} / H\right) \delta l=\delta W
$$

and this may be transformed to

$$
q_{1} \delta Q_{1}+q_{2} \delta Q_{2}+\left(K^{2} / H\right) \delta l=\delta\left(Q_{1} q_{1}+Q_{2} q_{2}-W\right) .
$$

Clearly, the left side of this equation is a perfect differential and this has very important consequences: for if we consider $q_{i}$ and $K$ as functions of $Q_{1}, Q_{2}$ and $l$, as in equations (3), (7), then

$$
\partial q_{i} / \partial l \equiv\left(\mathrm{d} C_{i j} / \mathrm{d} l\right) Q_{j}=\partial\left(K^{2} / H\right) / \partial Q_{i} \equiv 2 K^{(i)} K^{(j)} Q_{j} / H .
$$

Since this holds for all values of $Q_{1}$ and $Q_{2}$, we have

$$
\mathrm{d} C_{i j}(l) / \mathrm{d} l=2 K^{(i)}(l) K^{(j)}(l) / H,
$$

and this is seen to be a generalization of Irwin's [2] relation between compliance variations with crack length and stress intensity factors. A similar result has been derived in a special case by Rice and Levy (equations (9)-(14) of Ref. [5]); their application involved deriving cross terms analogous to $C_{21}$, given $K^{(1)}$ and $K^{(2)}$.

Here our viewpoint is different: it is assumed that we know the intensity factor $K^{(1)}$ and displacement field $\mathbf{u}^{(1)}$ associated with load system 1 . This means that we also know $C_{21}$, as is clear from equation (4). Hence, we find that equation (11), written for $i=2$ and $j=1$, enables us to solve for $K^{(2)}$ solely from a knowledge of the solution for load 1 :

$$
K^{(2)}(l)=\frac{H}{2 K^{(1)}(l)} \frac{\mathrm{d} C_{21}(l)}{\mathrm{d} l} \equiv \frac{H}{2 K^{(1)}(l)}\left\{\int_{\Gamma} \mathbf{t}^{(2)} \cdot \frac{\partial \mathbf{u}^{(1)}}{\partial l} \mathrm{~d} \Gamma+\int_{A} \mathbf{f}^{(2)} \cdot \frac{\partial \mathbf{u}^{(1)}}{\partial l} \mathrm{~d} A\right\} .
$$

[Here we pause to recall that $\mathbf{u}^{(1)}$ will be non-unique to within rigid-body displacements and since these may be chosen arbitrarily for each crack length, $\partial \mathbf{u}^{(1)} / \partial \mathrm{l}$ must be considered similarly non-unique. This has no effect on equation (12) since load set 2 is self-equilibrating and hence does no work on a rigid motion of the body.]

\section{THE WEIGHT FUNCTION}

Now, it is obvious that the stress intensity factor for load system 2 can in no way depend on the particular load system represented by 1 . Hence the fusction

$$
\mathbf{h}^{(1)}=\frac{H}{2 K^{(1)}(l)} \frac{\partial \mathbf{u}^{(1)}(x, y, l)}{\partial l}
$$

which, following Bueckner [1], we refer to as the weight function, must be essentially independent of the nature of load system 1 . 
To study its uniqueness consider another load system denoted by 3 . This must give the same result for $K^{(2)}$ when substituted for 1 in equation (12) and thus

$$
\int_{\Gamma} \mathbf{t}^{(2)} \cdot\left[\mathbf{h}^{(1)}-\mathbf{h}^{(3)}\right] \mathrm{d} \Gamma+\int_{A} \mathbf{f}^{(2)} \cdot\left[\mathbf{h}^{(1)}-\mathbf{h}^{(3)}\right] \mathrm{d} A=0
$$

for all symmetrical self-equilibrating load systems 2 . Since both 1 and 3 correspond to deformation fields that are symmetrical about the crack line we must have

$$
h_{x}^{(i)}(x,-y)=h_{x}^{(i)}(x, y)+\omega^{(i)} y ; \quad h_{y}^{(i)}(x,-y)=-h_{y}^{(i)}(x, y)+\alpha^{(i)}+\omega^{(i)} x
$$

where $\alpha^{(i)}$ and $\omega^{(i)}$ depend at most on crack length and reflect the rigid motion indeterminacy of $\mathbf{u}^{(i)}$. We now require that equation (14) hold for: (a) a unit point force in the $y$ direction at an arbitrary point $(x, y)$, with a symmetrical equilibrating force at $(x,-y)$ and (b) a unit point force in the $x$ direction at an arbitrary point $(x, y)$, a unit point force in the $-x$ direction at $\left(x^{\prime}, y^{\prime}\right)$ and symmetrical forces at $(x,-y)$ and $\left(x^{\prime},-y^{\prime}\right)$. This is readily shown to imply that

$$
h_{x}^{(1)}-h_{x}^{(3)}=\lambda-\Omega y ; \quad h_{y}^{(1)}-h_{y}^{(3)}=\mu+\Omega x,
$$

where $\lambda, \mu$ and $\Omega$ are constants. Thus $\mathbf{h}^{(1)}=\mathbf{h}^{(3)}$ to within rigid-body motions which are, in any event, arbitrary and inconsequential.

We therefore conclude that for any symmetrical load system leading to stress intensity factor $K$ and displacement field $\mathbf{u}$, the function

$$
\mathbf{h}=\mathbf{h}(x, y, l)=\frac{H}{2 K} \frac{\partial \mathbf{u}}{\partial l}
$$

(the derivative being taken at fixed values of the applied loads) is a universal function for a cracked body of any given geometry and composition, regardless of the detailed way in which the body is loaded. Once $h$ is determined from the solution for any particular load system, the stress intensity factor induced by any other symmetrical load system $t$ and $f$ is, from equation (12),

$$
K=\int_{\Gamma} \mathbf{t} \cdot \mathbf{h} \mathrm{d} \Gamma+\int_{A} \mathbf{f} \cdot \mathbf{h} \mathrm{d} A .
$$

It should also be noted that once $K$ is known we may go back to equation (17) and, by integrating $\partial u / \partial l$ with respect to $l$, construct the entire displacement field provided it is known for one value of $l$ (say, $l=0$ ). Hence any one elastic crack solution is seen to contain a remarkable store of information. This information is most succinctly given through the weight function itself and it is of interest that the weight function may be determined directly in view of the following properties noted by Bueckner [1] and summarized briefly here:

Note that $\mathbf{h}$ satisfies the same differential equations as the displacement $\mathbf{u}$ and that when $\mathbf{h}$ is viewed as a displacement, the stresses which it produces require no body forces in $A$ or boundary surface forces on $\Gamma$ for their equilibration (this results, of course, since $\mathbf{h} \propto \partial \mathbf{u} / \partial l$ ). Ordinarily, these conditions would be interpreted as requiring that $\mathbf{h}$ be a rigid motion, since a state of zero stress satisfies the null loading conditions. However, the elastic uniqueness theorem applies to uniqueness on the class of crack tip displacement fields carrying bounded total energy and $\mathbf{h}$ is not of this class. The strongly singular part of h can be determined by recalling that the displacement fields to all (bounded energy) 
solutions for cracks take the form

$$
\mathbf{u}=H^{-1} K r^{\frac{1}{2}} \mathbf{g}(\theta)+\ldots
$$

where the dots stand for terms resulting in non-singular stresses, where $r \theta$ are polar coordinates centered at the crack tip and where $\mathbf{g}$ is a universal function of $\theta$ (and also of ratios of elastic moduli) [2-4].

Since $\partial r / \partial I=-\cos \theta$ and $\partial \theta / \partial l=\sin \theta / r$, where $\theta=0$ along the line ahead of the crack, we have

$$
\partial \mathbf{u} / \partial I=H^{-1} K r^{-\frac{1}{2}}[(\sin \theta)(\mathrm{dg} / \mathrm{d} \theta)-(\cos \theta)(\mathrm{g} / 2)]+\ldots
$$

where now the dots represent all terms which are bounded at the tip and which, if taken individually, would correspond to bounded total energy. Hence we see from equation (17) that

$$
\mathbf{h}=r^{-\frac{1}{2}}[2(\sin \theta)(\mathrm{d} \mathbf{g} / \mathrm{d} \theta)-(\cos \theta) \mathbf{g}] / 4+\mathbf{h}^{*}
$$

where $h^{*}$ is a displacement field of the usual bounded energy class for an elastic crack problem.

From this point of view it is easy to see that $h$ is a universal function for a given geometry and composition : its strongly singular $\left(r^{-\frac{1}{2}}\right)$ part is universal and $\mathbf{h}^{*}$ is chosen so that it, together with the prescribed $r^{-\frac{1}{2}}$ term, results in zero surface tractions on $\Gamma$ and zero body forces in $A$. Since the $r^{-\frac{1}{2}}$ term creates no tractions on the crack surfaces, the problem of determining $\mathbf{h}^{*}$ falls into the class of bounded energy problems for which there is uniqueness. Clearly, the result for $h$ bears no relation to any particular load system to which the body may be subjected.

An example of the method follows. Also, a three-dimensional theory for a weight function is developed in the Appendix, although this theory appears at present to be less directly useful in the determination of stress intensity factors.

\section{AN EXAMPLE}

To illustrate the procedure let us follow Bueckner [1] in considering the Inglis problem of a crack of length $l$ in an infinite body subject to a remotely uniform tensile stress $\sigma$. We take the origin of the $x y$ coordinate system at one tip of the crack, the other being at $(l, 0)$, so that the $y$ displacements along the upper and lower crack surfaces $(y= \pm 0)$ and the stress intensity factor are

$$
u_{y}= \pm 2 \sigma x^{\frac{1}{2}}(l-x)^{\frac{1}{2}} / H, \quad K=\sigma(\pi l / 2)^{\frac{1}{2}}
$$

where $H=E /\left(1-v^{2}\right)$ for plane strain. Thus

$$
\left.\begin{array}{c}
\partial u_{y} / \partial l= \pm \sigma x^{\frac{1}{2}}(l-x)^{-\frac{1}{2}} / H, \\
h_{y}(x, \pm 0, l)=(H / 2 K)\left(\partial u_{y} / \partial l\right)= \pm(2 \pi l)^{-\frac{1}{2}} x^{\frac{1}{2}}(l-x)^{-\frac{1}{2}},
\end{array}\right\}
$$

and

from equation (17). We may therefore employ equation (18) to write the expression for $K$ at the $(l, 0)$ crack tip due to a traction distribution $t_{y}= \pm p(x)$ along the surfaces of the 
crack and the result is

$$
K=\left(\frac{2}{\pi l}\right)^{\frac{1}{2}} \int_{0}^{l} p(x)\left(\frac{x}{l-x}\right)^{\frac{1}{2}} \mathrm{~d} x .
$$

This checks with known results (e.g. [3]) derived independently through Muskhelishvili's analytic function methods.

All problems of symmetrical loading may be reduced, by superposition of a solution for a crack-free body under the same loads, to a similar problem of prescribed normal tractions along the crack. Hence it would seem advisable that, to the extent possible, displacements of the crack surfaces [or better the weight function $h_{y}(x, \pm 0, l)$ ] as well as stress intensity factors be reported when crack problems are solved. Bueckner [1], for example, shows how his previous solution for the edge-cracked half-plane in tension may be employed to obtain the weight function for that case.

It is also worthy of note that the weight function at points remote from the crack tip could be determined with great accuracy by finite difference or finite element methods applied to the determination of $\mathbf{h}^{*}$ in equation (21). The inaccuracy of such methods near the tip would then be irrelevant. This procedure seems, in fact, to be closely related to that proposed by Barone and Robinson [6] for numerical determination of coefficients in eigenfunction expansions of stress fields about corner singularities.

Acknowledgement-This study was supported by the National Aeronautics and Space Administration under Grant NGL-40-002-080 to Brown University.

\section{REFERENCES}

[1] H. F. BueCKNeR, A novel principle for the computation of stress intensity factors. Z. angew. Math. Mech. 50, $529-546(1970)$.

[2] G. R. IRwIN, Fracture Mechanics, in Structural Mechanics, édited by J. N. Goodier and N. J. Hoff, pp. 557591. Pergamon Press (1960).

[3] J. R. RICE, Mathematical Analysis in the Mechanics of Fracture, in Fracture, edited by H. LIEBowIrz, Vol. II, pp. 191-311. Academic Press (1968).

[4] G. C. SiH, P. C. PARIS and G. R. IRwin, On cracks in rectilinearly anisotropic bodies. Int. J. Fracture Mech. 1, 189-203 (1965).

[5] J. R. RICE and N. Levy, The part-through surface crack in an elastic plate. J. Appl. Mech. in press.

[6] M. R. BARONE and A. R. RoBinson, Approximate Determination of Stresses near Notches and Corners in Elastic Media by an Integral Equation Method, University of Illinois Civil Engineering Studies, Structural Research Series No. 374 (1971).

\section{APPENDIX}

\section{Three-dimensional bodies}

An analogous theory may be developed for three-dimensional crack problems : let $V$ be the volume and $S$ the bounding surface of a body containing a planar crack, with both the body and all load systems under consideration being symmetrical about the plane of the crack. The contour lying along the tip of the crack is denoted by $L$; this is assumed smooth. Generalized forces $Q_{1}$ and $Q_{2}$ are defined so that, for example,

$$
\mathbf{t}=Q_{1} \mathbf{t}^{(1)} \text { on } S \text { and } \mathbf{f}=Q_{1} \mathbf{f}^{(1)} \text { in } V
$$


for load system 1 . Associated generalized displacements $q_{1}$ and $q_{2}$ may be defined through replacing $\Gamma$ by $S$ and $A$ by $V$ in equation (2). Further, equations (3) and (4) may be written with these same replacements when both load systems act simultaneously.

We shall wish to characterize energy changes when the crack surface is advanced by an infinitesimal amount $\delta l$, where $\delta l$ is a smooth function of position along $L$ marking the advance of the crack in a direction locally normal to $L$. The notation $\delta_{l}(\ldots)$ will denote the first order variation in the quantity $(.$.$) , viewed as a function of crack position and$ some other variables, when only the crack position is varied. Thus we write in analogy to equation (6)

$$
\left(\delta_{l} W\right)_{\text {fixed displ. }}=-\int_{L}\left[\left(K^{2} / H\right) \delta l\right] \mathrm{d} L,
$$

where $W$ is the total strain energy of the body and $H$ has its plane strain value. For simultaneous action of both load systems,

$$
K=Q_{1} K^{(1)}+Q_{2} K^{(2)}
$$

where $K^{(1)}$ and $K^{(2)}$ are functions of position along $L$ the first assumed known and the latter to be found.

Since $\partial W / \partial q_{i}=Q_{i}$, a general variation in the strain energy may be written as

$$
Q_{1} \delta q_{1}+Q_{2} \delta q_{2}-\int_{L}\left[\left(K^{2} / H\right) \delta l\right] \mathrm{d} L=\delta W
$$

and if we rearrange this in analogy to equation (9), viewing $q_{i}$ and $K$ as being dependent on $Q_{i}$ and the crack position, then we may write in analogy to equation (10) that

$$
\delta_{l} q_{i} \equiv\left(\delta_{l} C_{i j}\right) Q_{j}=\int_{L}\left[\frac{\partial}{\partial Q_{i}}\left(\frac{K^{2}}{H}\right) \delta l\right] \mathrm{d} L \equiv \int_{L} \frac{2}{H}\left[K^{(i)} K^{(j)} Q_{j} \delta l\right] \mathrm{d} L .
$$

Thus the three-dimensional version of the relationship between compliance variations and stress intensity factors is

$$
\delta_{l} C_{i j}=\int_{L} \frac{2}{H}\left[K^{(i)} K^{(j)} \delta l\right] \mathrm{d} L
$$

When $i=2$ and $j=1$ this becomes, in analogy to equation (12),

$$
\int_{L} \frac{2}{H}\left[K^{(1)} K^{(2)} \delta l\right] \mathrm{d} L=\int_{S} \mathbf{t}^{(2)} \cdot \delta_{l} \mathbf{u}^{(1)} \mathrm{d} S+\int_{V} \mathbf{f}^{(2)} \cdot \delta_{l} \mathbf{u}^{(1)} \mathrm{d} V
$$

and this allows a (rather complete) knowledge of the solution for load system 1 to serve as a basis for determining the stress intensity factor for load system 2 .

The difficulty is, of course, that three-dimensional solutions for any load system 1 will not be known with such complete generality that the first order variation $\delta_{l} \mathbf{u}^{(1)}$ can be determined for completely arbitrary variations $\delta l$ along $L$. If the result were known, say as an equation of the form

$$
\delta_{l} \mathbf{u}^{(1)}(P)=\int_{L}\left[U^{(1)}\left(P, P^{\prime}\right) \delta l\left(P^{\prime}\right)\right] \mathrm{d} L\left(P^{\prime}\right)
$$


where $P$ denotes a general point of the body and $P^{\prime}$ a point along the crack tip, then we could solve for $K^{(2)}$ at any point $P^{\prime}$ along $L$ as

$$
K^{(2)}\left(P^{\prime}\right)=\frac{H\left(P^{\prime}\right)}{2 K^{(1)}\left(P^{\prime}\right)}\left\{\int_{S} \mathbf{t}^{(2)}(P) \cdot U^{(1)}\left(P, P^{\prime}\right) \mathrm{d} S(P)+\int_{V} \mathbf{f}^{(2)}(P) \cdot U^{(1)}\left(P, P^{\prime}\right) \mathrm{d} V(P)\right\}
$$

which is the general three-dimensional version of equation (12).

In similar fashion, a three dimensional weight function may be defined as

$$
\mathbf{h}\left(P, P^{\prime}\right)=H\left(P^{\prime}\right) \mathbf{U}^{(1)}\left(P, P^{\prime}\right) /\left[2 K^{(1)}\left(P^{\prime}\right)\right]
$$

and this is a unique (to within rigid motions) function of $P$ and $P^{\prime}$ for a given crack geometry in a body of given overall geometry and composition, being completely independent of the way in which the body is loaded. If it is determined from the solution for any particular load system, then the solution for $K$ at $P^{\prime}$ induced by any other load system $t$ and $f$ may be obtained from

$$
K\left(P^{\prime}\right)=\int_{S} \mathbf{t}(P) \cdot \mathbf{h}\left(P, P^{\prime}\right) \mathrm{d} S(P)+\int_{V} \mathbf{f}(P) \cdot \mathbf{h}\left(P, P^{\prime}\right) \mathrm{d} V(P) .
$$

Of course, there will exist cases for which knowledge of an integrated average of the intensity factor, as in equation (A7), is sufficient and this presents less stringent requirements as to the generality in which $\delta_{l} \mathbf{u}^{(1)}$ must be known. An example is the case of a pennyshaped crack in an axially symmetric body. Then knowledge of the solution, as a function of crack radius, for any one axially symmetric load system would allow in an obvious way for the determination of the intensity factor for any other axially symmetric load system.

(Received 18 June 1971 ; revised 9 November 1971)

\begin{abstract}
Аб̆стрвкт-Оказывается, что если известны поле перемещений и фактор интенсивности напряжений, в качестве функций длины шели, для любой симметрической системы нагрузки, действуюшей на линейное упругое тело в пиоском дефррмационном состоянии, тогда можно непосредственно определить фактор интенсивности напряжений для другой какой либо симметрической системы нагрузки, действующей на тоже самое тело. Решение тесно связанр с "функцией веса" ьюкнера /1/, вследствие которой представляется фактор интенсивности напряжений в виде суммы похожих работе пройзведений из приложенных усилий и значений функции веса в точках их приложения. Дается пример зтого метода, в котором используется решения для шели под влиянием отдаленного однородного поля напряжений, для обобщения выражений фактора интенсивности напряжений, вследствие произвольного распределения тяговых усилий на поверхностях щели. В приложении, определяется соответсвующая теория для трехмерных задая шели, хотя зто оказывается непосредствекно полезным, главным образом, для задач с осевой симметрией.
\end{abstract}

